\title{
Perspectiva del Odontólogo sobre la necesidad de unir la Logopedia a la Práctica Clínica
}

\author{
Daniel Antunes Freitas ${ }^{a}$, Stéffany Lara Nunes Oliveira Antunes ${ }^{b}$, \\ Luis Fang Mercado ${ }^{c}$, Alejandra Herrera Herrerac ${ }^{c}$, Antonio Díaz Caballero ${ }^{d}$
}

a Odontólogo UNINCOR, Especialista en Bioética UFLA, Magister en Odontología UNINCOR, Docente titular Facultades Unidas do Norte de Minas, FUNORTE, Montes Claros, MG, Brasil.

' Estudiante Facultad de Logopedia, Facultades Unidas do Norte de Minas, FUNORTE, Montes Claros, MG, Brasil.

c Odontólogo y Joven investigador del Grupo de investigaciones GITOUC, Facultad de Odontología, Universidad de Cartagena.

d Odontólogo, Universidad de Cartagena. Especialista en Periodoncia Universidad Javeriana. Magister en Educación, Universidad del Norte, Estudiante de Doctorado en Ciencias Biomédicas, Universidad de Cartagena, Docente titular Universidad de Cartagena, Director Grupo de investigaciones GITOUC.

Correspondencia: Luis Fang Mercado, Facultad de Odontología, Universidad de Cartagena, Campus de la Salud, Barrio Zaragocilla, Cartagena, Bolívar, Colombia. Telf.: $057+5+6698172$ ext. 110 , correo electrónico: luifang87@ gmail.com, adiazc1@ unicartagena.edu.co

Recibido el 13 de octubre de 2010.

Aceptado para su publicación el 7 de diciembre de 2010 .

\section{RESUMEN}

Objetivo. Analizar la perspectiva de los odontólogos generales y especialistas sobre la necesidad de unir la logopedia a la práctica de odontología.

Diseño. Estudio observacional, descriptivo y de corte transversal.

Emplazamiento. Escuela de Odontología de FUNORTE, Montes Claros-Brasil.

Participantes. Odontólogos generales, ortodoncistas, odontopediatras y ortopedas faciales. Mediciones principales. Edad, sexo, nivel académico, experiencia laboral, experiencia de remisión de los participantes, motivos de remisión, posición que adoptan los pacientes al ser remitidos.

Resultados. $\mathrm{N}=73$ (tasa de respuesta del 100\%), el 100\% de los encuestados considera importante unir la logopeda a la práctica clínica. El $74 \%$ de los individuos tiene experiencia de remisión, de estos el $51,9 \%$ refiriere que algunas veces los pacientes necesitan asistencia del logopeda, motivados en un $31,5 \%$ de los casos por respiración oral u oronasal, los cuales son remitidos después de finalizar el tratamiento según lo dicho por el $55,6 \%$ de los encuestados. Sin embargo, el 53,7 \% reportó que los pacientes rara vez asisten a la consulta de este especialista, desmotivados posiblemente por condiciones económicas desfavorables según los dicho por el $72,2 \%$ de los participantes. Solo el $11 \%$ de los individuos con experiencia laboral trabaja interdisciplinariamente en el mismo lugar de trabajo con un logopeda. Del mismo modo, se considera que se aumenta la aceptación de los tratamientos por parte de los pacientes cuando el trabajo conjunto con el logopeda se hace evidente.

Conclusión. Se observó que los encuestados consideran importante la atención del logopeda conjunta a su profesión.

Palabras clave. Fonoaudiología, Odontología, Grupo de Salud Interdisciplinario.

\section{ABSTRACT}

Dentists' opinion on the need to link speech therapy with dental practice

Objective. To determine the opinion of dentists and dental specialists on the need to link speech therapy with the practice of dentistry.

Design. Observational, descriptive and cross-sectional.

Setting. FUNORTE School of Dentistry, Montes Claros, Brazil.

Participants. General dentists, orthodontists, paediatric dentists and facial orthopaedics specialists.

Mean Measurements. Age, gender, educational level, work experience, referral experience of participants, reasons for referral, the position taken by patients being referred.

Results. $n=73$ (response rate $100 \%$ ), $100 \%$ of respondents considered it important to link speech therapy with dental practice. Seventy four percent of participants had experience with referrals and $51.9 \%$ of these reported that their patients sometimes needed assistance from speech therapists. In $31.5 \%$ of cases this was due to oral or oronasal breathing. $55.6 \%$ of the participants reported that patients are referred to a speech therapist at the end of their treatment. However, $53.7 \%$ reported that patients rarely attend the specialist visit and according to $72.2 \%$ of participants this is probably because of the patient's financial situation. Only $11 \%$ of the participants had worked in an inter-disciplinary setting with a speech therapist. Similarly they considered that patient acceptance of treatment is increased when working with a speech therapist.

Conclusion. It was concluded that the respondents considered that linking speech therapy with their profession was important.

Key words. Speech, Language and Hearing Sciences, Dentistry, Interdisciplinary Health Team. 


\section{INTRODUCCIÓN}

Al iniciar la práctica clínica todo profesional de la salud asume responsabilidades ante la sociedad, los pacientes y colegas, sin embargo la eficacia de los tratamientos a menudo dependen del trabajo conjunto entre las distintas disciplinas del conocimiento científico ${ }^{1}$. La relación entre la logopedia y la odontología, en especial la ortodoncia, se basa en el estudio de las funciones orales reflejo-vegetativas de carácter pre-lingüístico como la respiración, succión, masticación y deglución, las cuales permiten crear un vínculo entre estas dos profesiones. En el caso de la odontología estas funciones son responsables de la correcta estabilidad e inclinación axial de los dientes y morfología maxilar².

El sistema estomatognático presenta cierta complejidad y a su vez se relaciona con muchas funciones corporales de gran vitalidad. Muchos factores contribuyen a la estabilidad de las estructuras óseas y patrones miofuncionales de la cara y para su estudio se deben abarcar diversas áreas del conocimiento donde intervienen diferentes profesiones de la salud ${ }^{3}$, determinando así un buen crecimiento facial. La relación entre los distintos profesionales es extremadamente importante no sólo para los ortodoncistas, odontólogos y ortopedistas faciales, sino también para el clínico y los especialistas que trabajan con una visión global y ven al paciente como un ser complejo en el que muchos aspectos, incluida la emoción, pueden provocar problemas físicos y/o funcionales. Varios trastornos pueden afectar el sistema estomatognático en mayor 0 menor grado, en sus funciones o estructuras. El logopeda es un profesional que debe formar parte del equipo interdisciplinario ${ }^{4}$.

En la última década la logopedia, en particular en el ámbito de la motricidad oral, creció hasta tornarse más científica, realizando un trabajo más personalizado, y por lo tanto el logro de mejores resultados en menos tiempo. Todos estos avances son el producto del estudio de los campos afines con una mejor comprensión de las alteraciones oromiofuncionales. El logopeda pasó a tener una visión más amplia de la situación y así relacionar la forma y función. Se entiende que sin este análisis el diagnóstico puede ser totalmente contradictorio, lo que resulta en la frustración al final del tratamiento, por ejemplo la función se puede modificar y aun así no presentarse cambios en la forma; en tales casos, el trabajo funcional se convierte en vano ${ }^{5-7}$.

El objetivo de este estudio fue analizar la perspectiva de los odontólogos generales y especialistas docentes de la Escuela de Odontología de las Facultades Unidas del Norte de Minas - FUNORTE en la ciudad de Montes Claros - MG, Brasil, sobre la necesidad de unir la logopedia a la práctica odontológica, además de llamar la atención sobre la importancia del trabajo interdisciplinario entre la odontología y logopedia, buscando siempre el equilibrio del sistema estomatognático y la promoción de la salud integral y armónica de los pacientes.

\section{MATERIAL Y MÉTODO}

Se realizó un estudio observacional, descriptivo y

\begin{tabular}{c|c|c}
\hline Características & $\begin{array}{c}\text { Frecuencia } \\
\mathbf{n = 7 3}\end{array}$ & $\mathbf{( \% )}$ \\
\hline Sexo $\quad$ Hombres & 34 & 46,6 \\
$-\quad$ Mujeres & 39 & 53,4 \\
Título académico & & \\
$-\quad$ Odontólogo General & 13 & 17,8 \\
$-\quad$ Ortodoncista & 21 & 28,8 \\
$-\quad$ Odontopediatra & 24 & 32,9 \\
Ortopedista Facial & 15 & 20,5 \\
\hline & Media & Desviación \\
Estándar \\
\hline Edad (años) & 35,0 & 1,25 \\
Experiencia Laboral (años) & 11,8 & 2,33 \\
Actividad Laboral (Horas diarias) & 9,19 & 1,19 \\
Universidad (Horas diarias) & 5,19 & 0,89 \\
Práctica Privada (Horas diarias) & 3,97 & 0,84 \\
\hline
\end{tabular}

Tabla 1. Datos generales y experiencia profesional de los participantes 


\begin{tabular}{c|c|c|c|c}
\hline \multirow{2}{*}{} & \multicolumn{2}{|c|}{ Sí remite } & \multicolumn{2}{c}{ No remite } \\
\cline { 2 - 5 } & $\begin{array}{c}\text { Frecuencia } \\
\mathbf{N = 5 4}\end{array}$ & $\begin{array}{c}\text { Porcentaje } \\
\mathbf{7 4 \%}\end{array}$ & $\begin{array}{c}\text { Frecuencia } \\
\mathbf{N}=\mathbf{1 9}\end{array}$ & $\begin{array}{c}\text { Porcentaje } \\
\mathbf{2 6 \%}\end{array}$ \\
\hline Título académico & & & & \\
- Ortodoncista & 23 & 31,5 & 1 & 1,4 \\
- Odontopediatra & 19 & 26,0 & 2 & 2,7 \\
- Ortopeda Facial & 12 & 16,4 & 3 & 4,1 \\
- Odontólogo General & 0 & 0,0 & 13 & 17,8 \\
\hline
\end{tabular}

Tabla 2. Distribución del nivel académico según historial de remisión

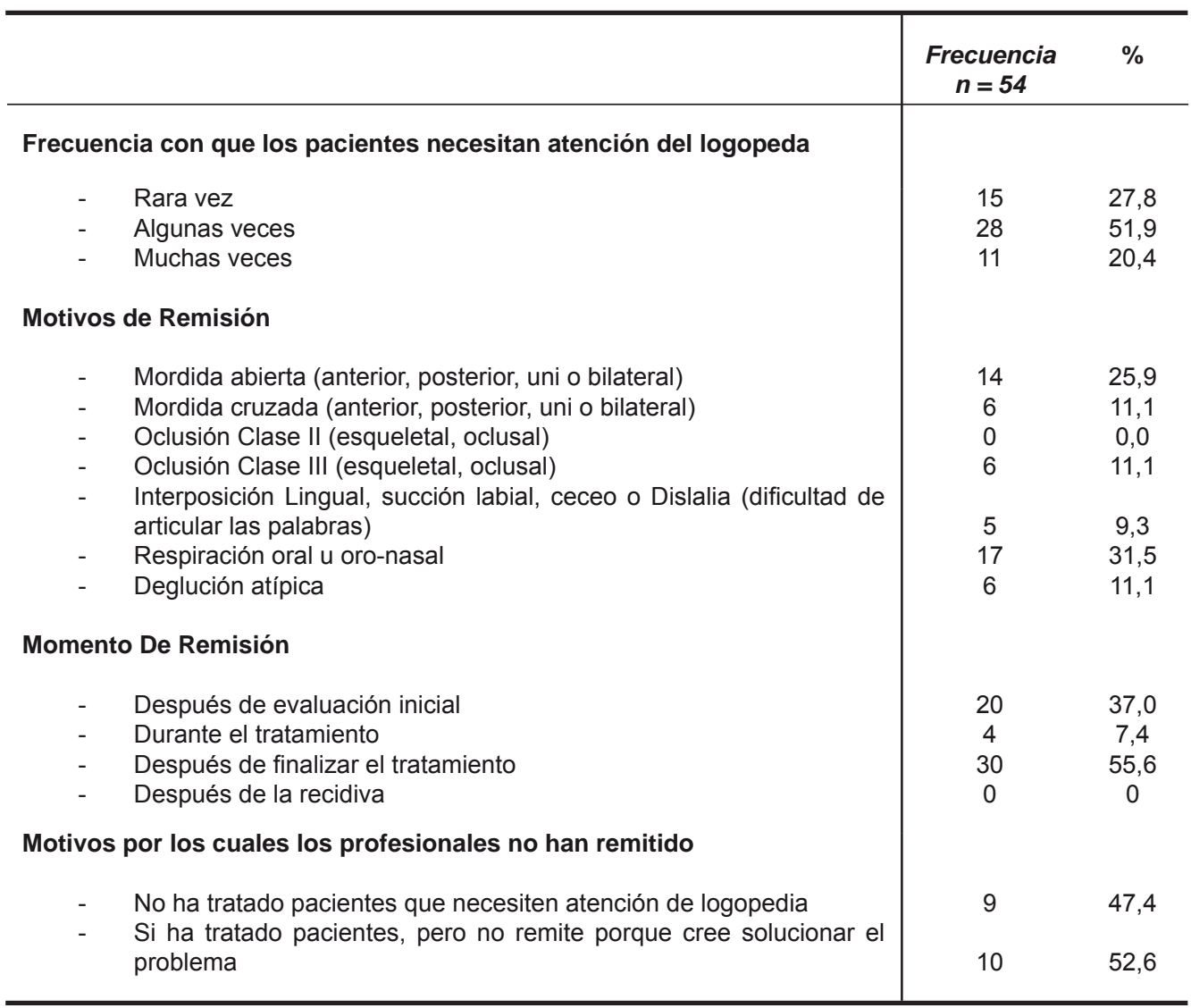

Tabla 3. Experiencia de remisión.

de corte transversal en la Escuela de Odontología de las Facultades Unidas del Norte de Minas FUNORTE en la ciudad de Montes Claros - MG Brasil. La población de estudio estuvo constituida por odontólogos generales y especialistas que imparten enseñanza en dicha institución. El tamaño de la muestra se calculó a través de la formula estadística para población finita basado en una prevalencia esperada del fenómeno de $95 \%{ }^{8}$, un nivel de confianza del 95\% y un margen de error tipo I del $5 \%$, con lo cual se obtuvo 73 sujetos. Se estableció un muestreo probabilístico con el método 


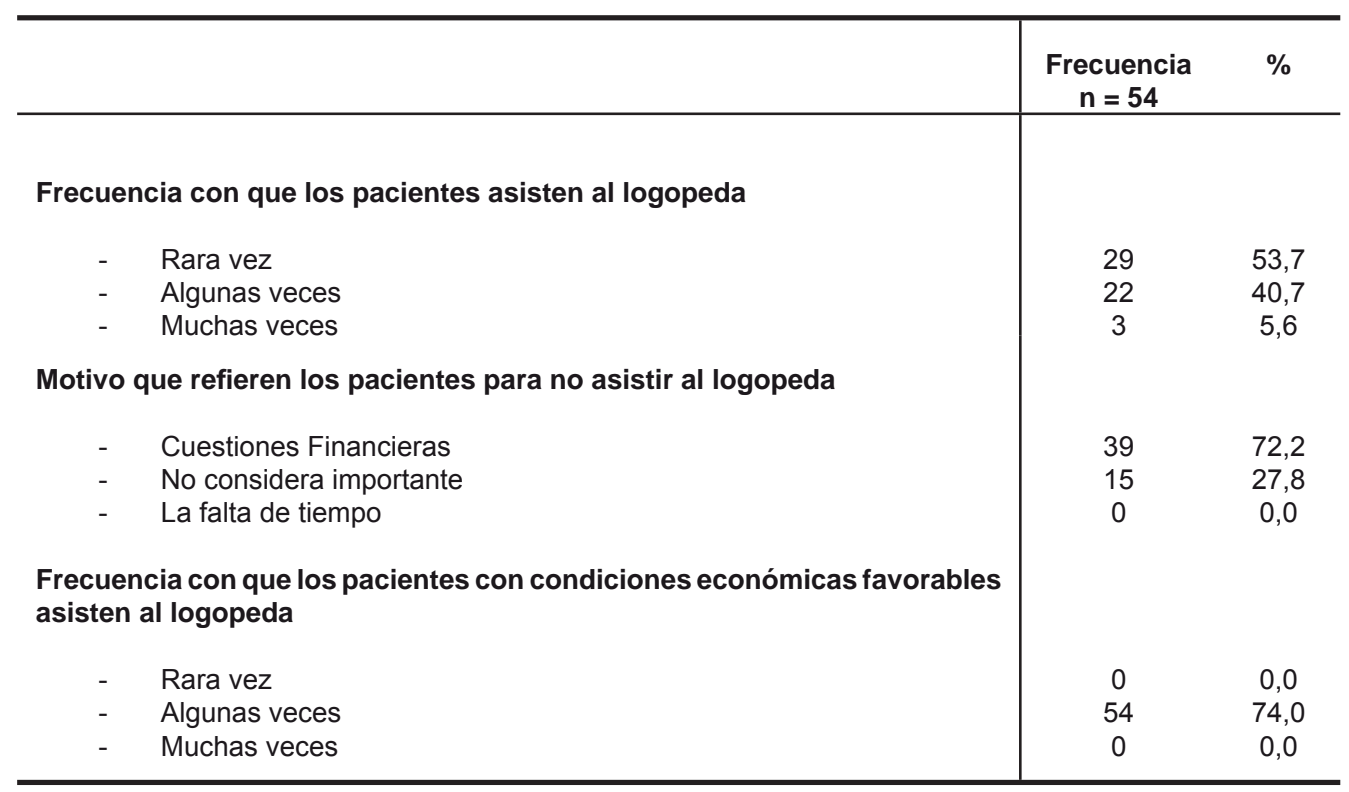

Tabla 4. Asistencia de los pacientes al logopeda.

\begin{tabular}{|c|c|c|}
\hline & $\begin{array}{c}\text { Frecuencia } \\
n=73\end{array}$ & $\%$ \\
\hline \multicolumn{3}{|c|}{ Logopeda de confianza al cual remitir a los pacientes } \\
\hline$-\quad \mathrm{Si}$ & 54 & 74,0 \\
\hline$-\quad$ No & 19 & 26,0 \\
\hline \multicolumn{3}{|c|}{ Presencia del logopeda en el grupo o lugar de trabajo } \\
\hline$-\quad \mathrm{Si}$ & 8 & 11,0 \\
\hline$-\quad$ No & 65 & 89,0 \\
\hline \multicolumn{3}{|c|}{$\begin{array}{l}\text { Aceptación del tratamiento y bienestar de los pacientes a partir de la } \\
\text { asistencia conjunta de un logopeda }\end{array}$} \\
\hline$-\quad \mathrm{Si}$ & 54 & 74,0 \\
\hline$-\quad$ No & 19 & 26,0 \\
\hline \multicolumn{3}{|c|}{ Importancia de unir la logopedia a la práctica clínica } \\
\hline$-\quad S i$ & 73 & 100 \\
\hline
\end{tabular}

Tabla 5. Importancia del logopeda en la práctica odontológica.

aleatorio simple, a partir de una lista completa de la población, en la cual se le asignó un código a cada individuo y finalmente, mediante una tabla de números aleatorios en Excel, se seleccionaron los sujetos que formaron parte de la muestra.

Previamente a la selección se tuvieron en cuenta los siguientes criterios: ser odontólogo general, ortodoncista, odontopediatra u ortopedista facial graduado, ser docente titular en la Escuela de Odontología de FUNORTE y ejercer la profesión en la práctica privada.

Para la recolección de información inicialmente se diseñó un instrumento tipo encuesta compuesta por 19 preguntas de selección múltiple con única 
respuesta, dentro de las cuales algunas preguntas fueron diseñadas para que fueran resueltas exclusivamente por aquellos participantes que no presentaron antecedentes de remisión y por aquellos que si remiten a sus pacientes al logopeda, e igualmente se desarrollaron interrogantes que debían ser contestados por todos los individuos. Esta encuesta evaluó principalmente la importancia de unir la logopedia a la práctica odontológica, seguida de la experiencia de remisión al logopeda por parte de los participantes, la posición que suelen adoptar los pacientes al ser remitidos, además de ciertas características de los individuos relacionadas con su experiencia laboral, nivel académico y tiempo de trabajo diario. Para el inicio de la recolección de los datos se facilitó el abordaje de los participantes a través de un diálogo directo con los investigadores, con la presentación personal de los mismos y la explicación de los objetivos de la investigación a través de un consentimiento informado por escrito. Además, se explicó la utilidad directa para los beneficiarios y la falta de riesgo a que estarían expuestos. Este estudio fue avalado por el comité de ética institucional, catalogado sin riesgo a partir de las consideraciones emanadas por la resolución 0135 de 2009 de FUNORTE.

Los evaluadores corroboraron el diligenciamiento completo de la encuesta por parte de los participantes en el momento en que éstos la estaban desarrollando. La información se registró en una tabla matriz diseñada en Microsoft Excel versión 2007. Para el análisis estadístico se utilizó el paquete SPSS STATISTICS versión 17.0 para Windows. El análisis univariado de tipo descriptivo de las variables cualitativas se expresó en frecuencias y proporciones, y de las variables cuantitativas como media y desviación estándar $( \pm \mathrm{DE})$. Posteriormente se desarrolló el análisis bivariado representado en tablas de contingencia usando pruebas de $\mathrm{X}^{2}$ de Pearson, siempre $\mathrm{y}$ cuando no hubiese valores esperados menores a 5 ; en caso de existir valores esperados menores a cinco 5 se empleó corrección de yates y test exacto de Fisher bajo una significación de 0.05 .

\section{RESULTADOS}

Se incluyó en el estudio un total de 73 profesionales, con una tasa de respuesta del $100 \%$. De acuerdo a los datos relacionados con la experiencia de remisión de los individuos, éstos se organizaron en dos grupos, uno correspondiente a aquellos profesionales que no remiten o no han remitido pacientes al logopeda y un segundo grupo concerniente a los individuos que si realizan remisiones al logopeda, en el cual se hace mayor énfasis. La información relacionada con resto de variables se analizó a partir de las respuestas del total de sujetos encuestados.

La población estudiada presentó una edad promedio de 35 años $\pm 1,25 \mathrm{DE}$, representada en un $53,4 \%$ por profesionales del sexo femenino. En promedio la experiencia profesional y laboral de los encuestados fue de 11,8 años $\pm 2,33 \mathrm{DE}$, siendo especialistas el $82,2 \%$ (tabla 1) y destacando, dentro de este grupo, los ortodoncistas con un 32,9 $\%$ (tabla 2).

Al indagar a los participantes sobre sus experiencias de remisión al logopeda, el $74 \%$ de los individuos han remitido o remiten a sus pacientes a este profesional de la salud. De este grupo destacan los ortodoncistas con un $31,5 \%$, siendo los especialistas con mayor historial de remisión, a diferencia de los odontólogos generales, quienes no reportaron algún antecedente de remisión de sus pacientes (tabla 2). Al realizar el análisis bivariado entre experiencia de remisión y el nivel académico de los encuestados, se encontró asociación estadísticamente significativa $(p<0,01)$ (IC 95\%: $0,16-0,61)$, donde los especialistas tienen mayor posibilidad de remitir a sus pacientes (tabla 6).

Conforme al reporte de los participantes que presentan experiencia de remisión, el 51,9\% de los individuos refirió que algunas veces los pacientes necesitan asistencia del logopeda, los cuales son remitidos después de finalizar el tratamiento según lo afirmado por el $55,6 \%$ de los encuestados, motivados en un $31,5 \%$ de los casos por respiración oral u oronasal. Aquellos profesionales que no tienen antecedentes de remisión al logopeda constituyen el $26 \%$ del total de los encuestados, quienes en un $52,6 \%$ de los casos no lo han hecho debido a que se han sentido capaces de solucionar por sus propios medios y conocimientos las afecciones estomatognáticas de sus pacientes sin necesidad de remitirlos (tabla 3 ).

De igual forma, acorde a lo referido por los encuestados, la posición que adoptan los pacientes en el momento de ser remitidos al logopeda es desalentadora, puesto que el 53,7 \% reportaron que los pacientes rara vez asisten a la consulta de este especialista, desmotivados posiblemente por condiciones económicas desfavorables según 
lo manifestado por el $72,2 \%$ de los participantes. Para el caso de aquellos pacientes con condiciones económicas favorables, la totalidad de los encuestados refirió que algunas veces estos asisten a la consulta del logopeda (tabla 4).

Al consultar la opinión y perspectiva de los odontólogos presentes en el estudio sobre unir la logopedia a la práctica clínica odontológica, éstos afirmaron en su totalidad sobre la necesidad de unir estas dos ramas de la salud. Al indagar si conocen algún logopeda de confianza, sólo los individuos con experiencia de remisión respondieron afirmativamente. De este grupo sólo el $11 \%$ labora interdisciplinariamente en el mismo lugar de trabajo con un logopeda. Del mismo modo, sólo aquellos con historial de remisión consideran que probablemente se puede aumentar la aceptación de los tratamientos por parte de los pacientes cuando el trabajo conjunto con el logopeda se hace evidente (tabla 5). Al realizar el análisis bivariado entre experiencia de remisión y conocimiento de un logopeda de confianza, resultó que no se asociaban entre sí ( $p>0,05)$ (IC 95\%: 0,19 - 2,42), sin embargo, al relacionar la experiencia de remisión con la variable que representa si la presencia de un logopeda en el mismo lugar o grupo de trabajo aumenta la aceptación de los tratamientos por parte de los pacientes y el bienestar propio de este, sí se encontró asociación ( $p>0,01)$, aunque con un amplio intervalo de confianza (IC 95\%: 2,42 25,73).

\section{DISCUSIÓN}

Para el desarrollo del presente estudio cabe resaltar que los resultados obtenidos corresponden sólo a la opinión y respuesta propia de odontólogos y especialistas afines pertenecientes a la muestra objeto de análisis, sin embargo se consideró una limitación al no poder conocer las apreciaciones de los pacientes propiamente remitidos por los profesionales encuestados. Para cubrir este inconveniente se tuvo en cuenta sólo la opinión de aquellos profesionales con experiencia en remitir a sus pacientes al logopeda y por lo tanto conocedores de la posición que adoptan éstos a la hora de ser remitidos. Otra limitación es la posibilidad de concluir relaciones entre variables cuando el estudio es puramente descriptivo, sin embargo se logró identificar asociaciones entre algunas de estas variables.

Antiguamente los odontólogos, en especial los ortodoncistas, en pocas ocasiones remitían a sus pacientes al logopeda para una evaluación clínica. En la actualidad es posible observar un gran número de pacientes remitidos de la clínica odontológica a terapia de audición y lenguaje ${ }^{9,10}$. La razón de estas remisiones es producto de una mayor concientización por parte del odontólogo y del logopeda en busca de los beneficios que trae consigo el trabajo conjunto de estas dos especialidades ${ }^{11,12}$. Los logopedas acompañan de forma sistemática los avances de la odontología al hacer énfasis en los conocimientos sobre crecimiento cráneofacial y sus alteraciones, por medio de estudios radiográficos y análisis cefalométricos, lo que le da importancia al tratamiento multidisciplinario ${ }^{13}$. Lo anterior se corrobora en este estudio, donde se hace evidente la superioridad de aquellos profesionales con experiencia de remisión, además de la importancia y necesidad del trabajo conjunto e interdisciplinario según las expresiones de los odontólogos encuestados.

La asociación entre ortodoncistas y logopedas es de suma importancia, debido a que la función que ejercen los aparatos ortodónticos u ortopédicos limitan o alteran la disposición de los arcos dentarios, mientras que la logopedia trabaja la rehabilitación miofuncional orofacial, que tiende a modificar las funciones alteradas ${ }^{14}$. Es importante destacar el papel del logopeda en el ajuste de la función del habla para que en ningún caso se produzca la recaída debido a la mala posición de la lengua después del tratamiento ortodóntico. Cabe anotar que los patrones de la deglución, masticación y respiración están relacionados con maloclusiones y alteraciones del habla ${ }^{15-17}$. La mitad de los encuestados con experiencia de remisión indicaron que sus pacientes requieren terapia del habla algunas veces, principalmente producto de alteraciones en la respiración, sin dejar de lado patrones de deglución u oclusión.

En cuanto a la frecuencia con que los pacientes buscan asistencia del logopeda, la mitad de los encuestados reportaron que éstos muy pocas veces lo hacen. Los estudios indican que hay cierta resistencia de los pacientes a realizar una evaluación de logopedia solicitada por los odontólogos ${ }^{18}$. Es posible que conocer a un especialista en el área de logopedia facilite e influya en la posibilidad de remitir a los pacientes que lo necesiten. Aunque en el presente estudio no existió asociación o relación entre estas dos variables, sí se observó que sólo aquellos profesionales con experiencia de remisión conocen a un logopeda, sin dejar de lado que ser especialista en ortodoncia, odontopediatría u 
ortopedia facial permite remitir significativamente a los pacientes a diferencia de un odontólogo general. De acuerdo a la literatura ${ }^{19}$, la proximidad física dentro de la misma área de trabajo entre los distintos profesionales de la salud es un factor importante para los odontólogos, ya que permite interactuar con otros profesionales. En esta investigación sólo un puñado de los encuestados trabaja en el mismo espacio con un logopeda, sin embargo todos aquellos participantes con historial de remisión consideran que la asistencia conjunta entre odontología y logopedia beneficia la comunicación de las dos áreas y en especial al paciente. Este mayor contacto, aunque quizás no en el mismo lugar de trabajo, puede contribuir a la normalización de la valoración clínica ${ }^{1}$ y la comprensión de los objetivos terapéuticos ${ }^{20}$.

Lamayoría de los encuestados remitea sus pacientes al logopeda después de culminar el tratamiento. La decisión de iniciar una terapia de logopedia, o si es esencial llevarla a cabo, se debe tomar conjuntamente entre los profesionales ${ }^{21}$, teniendo en cuenta que cada paciente tiene una necesidad diferente. El enfoque al comienzo del tratamiento de ortodoncia permite una mejor discusión de los casos, evitando intervenciones tardías. El consenso es que la corrección ortodóntica sólo puede llevarse a cabo adecuadamente si está en armonía con el equilibrio muscular del paciente. Por lo tanto, se debe esperar culminar la terapia con el logopeda para finalizar el tratamiento ortodóntico ${ }^{22,13}$.

La principal justificación de porqué los pacientes no acuden a consulta con el logopeda es el factor económico. Es posible que los pacientes no comprendan la importancia de conocer la opinión de otro profesional de la salud para el tratamiento de las alteraciones miofuncionales, y aunque esta hipótesis no esté confirmada es probable que las capacidades financieras según los entrevistados influyan en la toma de decisiones, como es el caso de los pacientes con condiciones financieras favorables, quienes sí se interesan en buscar atención del logopeda según esta investigación.

Por lo tanto, existe una amplia necesidad de difundir la actuación del logopeda en el campo de la motricidad orofacial, incluyendo las posibles limitaciones, debido a que el papel de este profesional no es muy bien definido por parte de algunos especialistas del área de la odontología ${ }^{24,25}$, quienes se muestran reacios a la hora de remitir a sus pacientes ${ }^{18,26}$, incluso en aquellos casos donde es necesaria la atención interdisciplinaria ${ }^{27}$. Por medio de este estudio se observó que los encuestados consideran importante la atención del logopeda conjunta a su profesión. Sin embargo, se pueden adelantar investigaciones semejantes que evalúen a los logopedas y a otros especialistas. Con esto se confirmaría o refutaría la necesidad de la interdisciplinariedad en la atención.

\section{BIBLIOGRAFÍA}

1. Korbmacher $\mathrm{H}$, Kahl-Nieke B. Optimizing interdisciplinary cooperation for patients with orofacial dysfunctions. Presentation of an interdisciplinary diagnostic referral sheet. J Orofac Orthop. 2001; 62(3):246-50.

2. Dahan J. Combined orthodontic-orthopedic approach: a second choice in some surgical cases. World $\mathrm{J}$ Orthod. 2008; 9(2):35-45.

3. Ray J. Effects of orofacial myofunctional therapy on speech intelligibility in individuals with persistent articulatory impairments. Int J Orofacial Myology. 2003; 29:5-14.

4. Landouzy JM, Sergent Delattre A, Fenart R, Delattre B, Claire J, Biecq M. The tongue: deglutition, orofacial functions and craniofacial growth. Int Orthod. 2009; 7(3):227-56.

5. Korbmacher HM, Schwan M, Berndsen S, Bull J, Kahl-Nieke $B$. Evaluation of a new concept of myofunctional therapy in children. Int J Orofacial Myology. 2004; 30:39-52.

6. Ray J. Orofacial myofunctional therapy in dysarthria: a study on speech intelligibility. Int J Orofacial Myology. 2002; 28:39-48.

7. Paul-Brown D, Clausen R. Collaborative approach for identifying and treating speech, language, and orofacial myofunctional disorders. Alpha Omegan. 1999; 92(2):3944.

8. Cup EH, Pieterse AJ, Knuijt S, Hendricks HT, van Engelen $B G$, Oostendorp RA, et al. Referral of patients with neuromuscular disease to occupational therapy, physical therapy and speech therapy: usual practice versus multidisciplinary advice. Disabil Rehabil. 2007; 29(9):71726.

9. Jung $\mathrm{MH}$. Evaluation of the effects of malocclusion and orthodontic treatment on self-esteem in an adolescent population. Am J Orthod Dentofacial Orthop. 2010; 138(2):160-6.

10. Lobbezoo F, van der Zaag J, Visscher CM, van der Meulen MJ, Becking AG, Naeije M. [Multidisciplinary diagnosis and treatment of craniomandibular disorders]. Ned Tijdschr Tandheelkd. 2000; 107(11):471-5.

11. Jaju $R$, Tate $A R$. The role of pediatric dentistry in multidisciplinary cleft palate teams at advanced pediatric dental residency programs. Pediatr Dent. 2009; 31(3):18892.

12. Hubalkova H, Holakovsky J, Brazda F, Diblik P, Mazanek J. Team approach in treatment of extensive maxillofacial defects - five case report serie. Prague Med Rep. 2010; 111(2):148-57.

13. Hurubeanu L, Baciut G, Zeilhofer HF, Sader R, Baciut M, Campian RS, et al. [New morpho-functional rehabilitation methods in cleft lip and palate]. Rev Med Chir Soc Med Nat lasi. 2008; 112(1):229-33.

14. Fujiki T, Inoue M, Miyawaki S, Nagasaki T, Tanimoto K, Takano-Yamamoto T. Relationship between maxillofacial morphology and deglutitive tongue movement in patients with anterior open bite. Am J Orthod Dentofacial Orthop. 2004; 125(2):160-7. 
15. Emerich K, Wojtaszek-Slominska A. Clinical practice. Later orthodontic complications caused by risk factors observed in the early years of life. Eur J Pediatr. 2009; 169(6):651-5.

16. Smithpeter J, Covell D, Jr. Relapse of anterior open bites treated with orthodontic appliances with and without orofacial myofunctional therapy. Am J Orthod Dentofacial Orthop. 2010; 137(5):605-14.

17. Burford D, Noar JH. The causes, diagnosis and treatment of anterior open bite. Dent Update. 2003; 30(5):235-41.

18. Doyle J. Initial consultations in hearing aid clinics in Australia. J Am Acad Audiol. 1994; 5(3):216-25.

19. Van Teeseling RA, Goene RJ. Interdisciplinary cooperation in dentistry. Ned Tijdschr Tandheelkd. 2003; 110(1):35-7.

20. Brown CR. Orofacial myofunctional disorders. Pract Periodontics Aesthet Dent. 1996; 8(7):698.

21. Austin AA, Druschel CM, Tyler MC, Romitti PA, West, II, Damiano PC, et al. Interdisciplinary craniofacial teams compared with individual providers: is orofacial cleft care more comprehensive and do parents perceive better outcomes? Cleft Palate Craniofac J. 2010; 47(1):1-8.
22. Pinsky TM, Goldberg HJ. Potential for clinical cooperation between dentistry and speech pathology. Int Dent J. 1977; 27(4):363-9.

23. Maciel CT, Leite IC. [Etiological aspects of anterior open bite and its implications to the oral functions]. Pro Fono. 2005; 17(3):293-302.

24. Melink S, Vagner MV, Hocevar-Boltezar I, Ovsenik M. Posterior crossbite in the deciduous dentition period, its relation with sucking habits, irregular orofacial functions, and otolaryngological findings. Am J Orthod Dentofacial Orthop. 2010; 138(1):32-40.

25. Costa JR, Pereira SR, Pignatari SS, Weckx LL. Posture and posterior crossbite in oral and nasal breathing children. Int $\mathrm{J}$ Orthod Milwaukee. 2010; 21(1):33-8.

26. Amaral E, Bacha S, Ghersel E, Rodrigues P. Inter-relação entre a odontologia ea fonoaudiologia na motricidade orofacial. Rev CEFAC. 2006; 8(3):337-51.

27. Cooper NS. Myofunctional therapy. Int J Orthod. 1973; 11(3):81-7. 